\title{
Efficacy of Alkaloids in Alleviating Myocardial Ischemia- Reperfusion Injury in Rats: A Meta-Analysis of Animal Studies
}

\author{
Shuai Wang $\mathbb{D}^{1,2}$ Han Liu $\mathbb{D}^{3},{ }^{3}$ Yang Zhang $\mathbb{D}^{2}$, and Liqun Ren $\mathbb{D}^{1}$ \\ ${ }^{1}$ Department of Experimental Pharmacology and Toxicology, School of Pharmaceutical Science, Jilin University, Changchun, \\ Jilin, China \\ ${ }^{2}$ Department of Vascular Surgery, The First Hospital of Jilin University, Changchun, Jilin, China \\ ${ }^{3}$ Department of Respiration, The First Hospital of Jilin University, Changchun, Jilin, China
}

Correspondence should be addressed to Liqun Ren; renlq@jlu.edu.cn

Received 25 October 2020; Accepted 9 March 2021; Published 19 March 2021

Academic Editor: Emilio Rojas del Castillo

Copyright (c) 2021 Shuai Wang et al. This is an open access article distributed under the Creative Commons Attribution License, which permits unrestricted use, distribution, and reproduction in any medium, provided the original work is properly cited.

\begin{abstract}
Background. Animal models are well established for studying the effects of alkaloids in preventing myocardial ischemia-reperfusion injury. However, few studies have investigated the therapeutic effects of alkaloids in humans. This meta-analysis and systematic review assessed the efficacy of alkaloids in attenuating infarct size in rats with myocardial ischemia-reperfusion injury. Methods. An integrated literature search including the PubMed, Embase, and Cochrane Library databases was performed to identify studies that evaluated the therapeutic effects of alkaloids on myocardial ischemia-reperfusion injury in rats. The main outcome was infarct size, and SYRCLE's risk of bias tool was used to assess the quality of the studies. Results. 22 studies were brought into the meta-analysis. Compared with the effects of vehicle, alkaloids significantly reduced infarct size (standardized mean difference $(\mathrm{SMD})=-0.45 ; 95 \%$ confidence interval $(\mathrm{CI})=-0.64$ to -0.26$)$. In subgroup analyses, isoquinoline alkaloids $(\mathrm{SMD}=-0.43 ; 95 \% \mathrm{CI}=-0.70$ to -0.16$)$ significantly reduced infarct size versus the control. Conclusion. Isoquinoline alkaloids can potentially alleviate myocardial ischemia-reperfusion injury. This meta-analysis and systematic review supply a reference for research programs aiming to develop alkaloid-based clinical drugs. This trial is registered with CRD42019135489.
\end{abstract}

\section{Introduction}

Myocardial infarction (MI) constitutes the main cause of morbidity and mortality in patients with coronary heart disease globally $[1,2]$. MI develops when there is a severe imbalance between myocardial oxygen supply and demand, resulting in myocardial hypoxia [1]. As the primary treatment, timely reperfusion plays a key role in the treatment of MI. However, a secondary cardiac injury called ischemiareperfusion (I/R) injury can increase myocardial infarct size and induce a series of pathologic reactions, such as arrhythmia, left ventricular remodeling, and cardiac dysfunction [3-5]. Therefore, it is extremely necessary to develop novel prevention and therapy strategies.

In recent years, some studies illustrated that natural compounds, such as alkaloids, flavonoids, and terpenoids, attenuate myocardial I/R injury [6-8]. Alkaloids comprise a group of naturally occurring chemical compounds that mostly contain basic nitrogen atoms [9]. With a large range of pharmacological effects, such as antitumor, antiatherosclerotic, anti-inflammatory, and anti-I/R injury activities [10-12], they have been widely used in traditional and modern medicine [9]. However, most studies on the attenuating effects of alkaloids on myocardial I/R injury focused on isoquinoline alkaloids such as berberine and coptisine [13, 14], and a consensus on the effects of other alkaloids on myocardial I/R injury has not been reached.

Animal studies are conducted to investigate the safety and efficacy of novel interventions. Moreover, they are extremely valuable to build a bridge between basic research and clinical trials. In general, the rat is the most popular animal model for evaluating myocardial I/R injury induced through ligation of the left anterior descending artery [15]. To date, most systematic reviews and meta-analyses evaluated the effects of alkaloids on myocardial infarct size in rat $\mathrm{I} / \mathrm{R}$ models in an effort to understand the clinical potential 
of alkaloids as anti-I/R injury agents. Thus, this systematic review and meta-analysis assessed the effects of alkaloids on infarct size in rats with myocardial I/R injury.

\section{Materials and Methods}

2.1. Reporting Standards. This systematic review conformed to the Preferred Reporting Items for Systematic Reviews and Meta-Analyses (PRISMA) statement, and the protocol was constructed using the SYRCLE format for animal intervention studies $[16,17]$.

2.2. Search Strategy. With the keywords "myocardial ischemia-reperfusion injury", "rats", and "alkaloid", an experienced literature seeker (SW) searched the PubMed, Embase, and Cochrane Library databases for studies published between January 2001 and June 2019. In addition, the following search strategies were employed: (myocardial infarction OR myocardial ischemia OR myocardial I/R) AND (rat OR rats) AND (alkaloid OR aporphine OR belladonna OR indole OR isoquinoline OR opiate OR quinolizidine OR xanthine). A manual checking was performed to identify relevant studies from the reference lists of the included studies and review articles. The search was conducted on June 12, 2019, and limited among the articles published in English language.

2.3. Inclusion and Exclusion Criteria. The inclusion criteria were as follows: (1) original research, (2) rat as the animal model, (3) myocardial I/R injury as the disease model, and (4) alkaloids as the intervention. The exclusion criteria were as follows: (1) case reports, conference abstracts, review articles, editorials, and comments; (2) missing data; and (3) overlapping or duplicate datasets.

2.4. Study Selection. Two reviewers (HL and YZ) independently searched the titles and abstracts of the articles identified by the literature search to select eligible studies. The full text of potentially relevant articles was retrieved and independently examined by two reviewers (SW and LR) to determine whether these studies were in accordance with the inclusion criteria. Disagreements on study selection were resolved by discussion and consensus.

2.5. Data Extraction. Data extraction was performed by two reviewers (SW and HL) independently from the eligible studies. The first author's name, year of publication, age of rats, gender, diet, weight, alkaloid dose, time and route of treatment, duration of ischemia and reperfusion, and control and treatment group sample sizes were examined. Data that were showed graphically in the original publications were extracted using Adobe Photoshop 7.0.

The main outcome was infarct size measured as a numerical or percentage value. Disagreements on data extraction were resolved by discussion and consensus.

2.6. Quality Assessment. Quality assessment of the included studies was conducted by two investigators (YZ and LR) independently using SYRCLE's risk of bias tool, which contains domains evaluating sequence generation, baseline char-

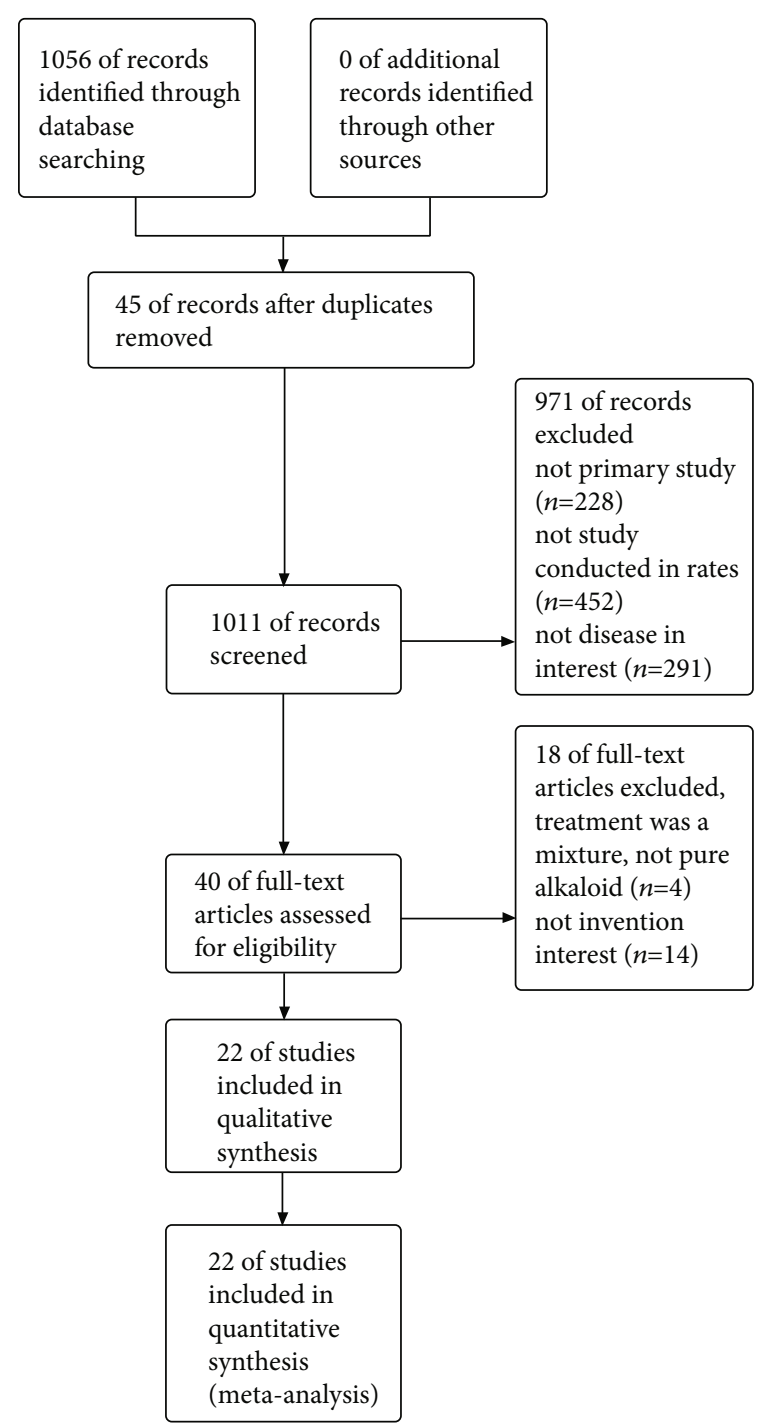

Figure 1: Flow diagram of the study identification and selection process.

acteristics, allocation concealment, random housing, blinding, random and selective outcome assessments, incomplete outcome data, and other sources of bias [18]. Publication bias was detected via visual inspection of funnel plots. Disagreements on quality assessment were resolved by discussion and consensus.

2.7. Data Synthesis and Statistical Analysis. Statistical analyses were conducted using Review Manager (RevMan Version 5.3 for Windows Copenhagen: The Nordic Cochrane Centre, The Cochrane Collaboration, 2014). Standardized mean differences (SMDs) with 95\% confidence intervals (CIs) were calculated to present the effects of vehicle and alkaloids on infarct size. A random-effects model was used to pool studies. Heterogeneity was categorized as moderate $\left(I^{2} \geq 30 \%\right)$ or high $\left(I^{2} \geq 50 \%\right)$ using the inconsistency index.

Multiple independent groups in a study (e.g., different alkaloid doses) were considered separate datasets. In nine studies [19-27], multiple groups investigating different 


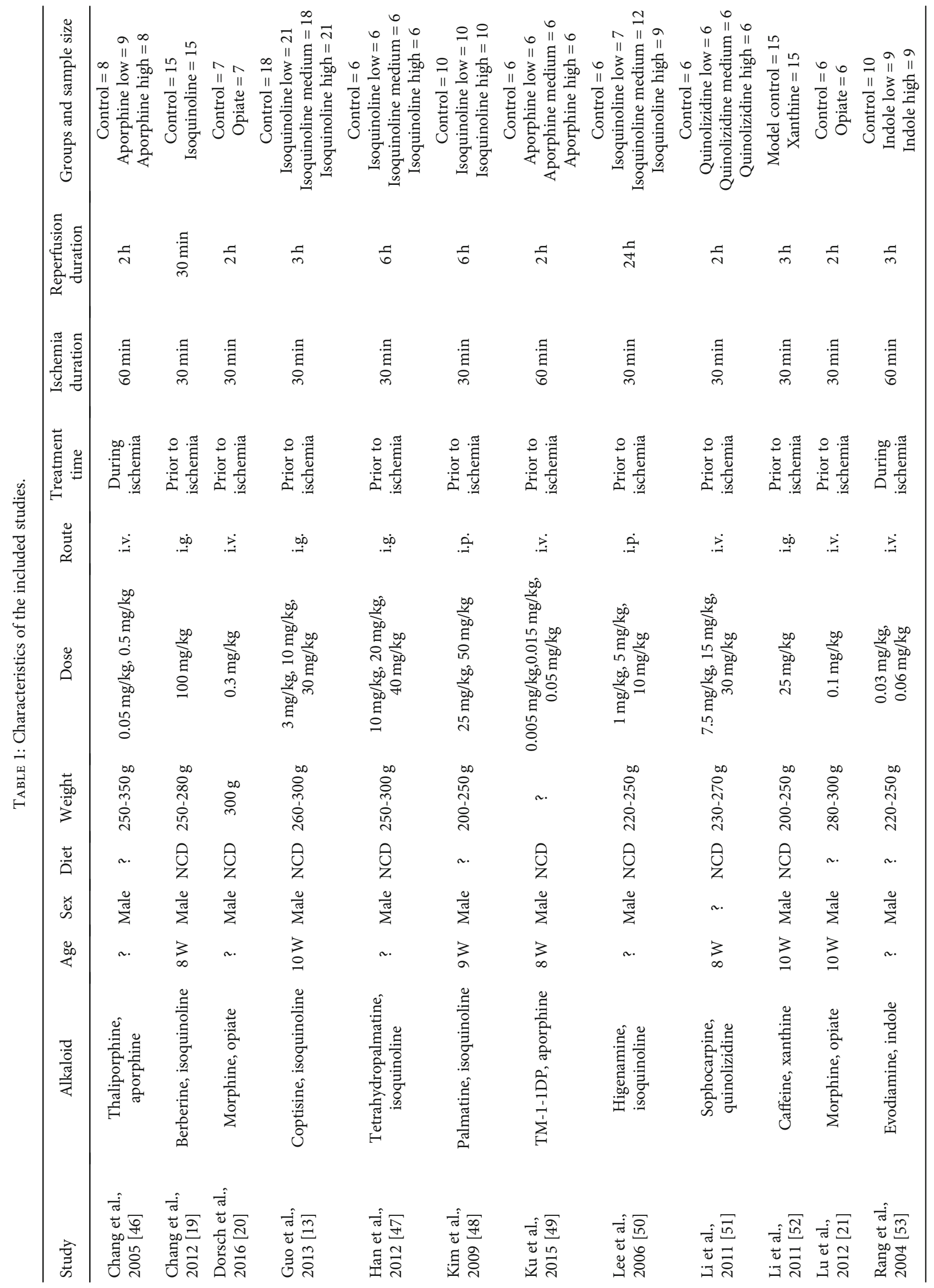




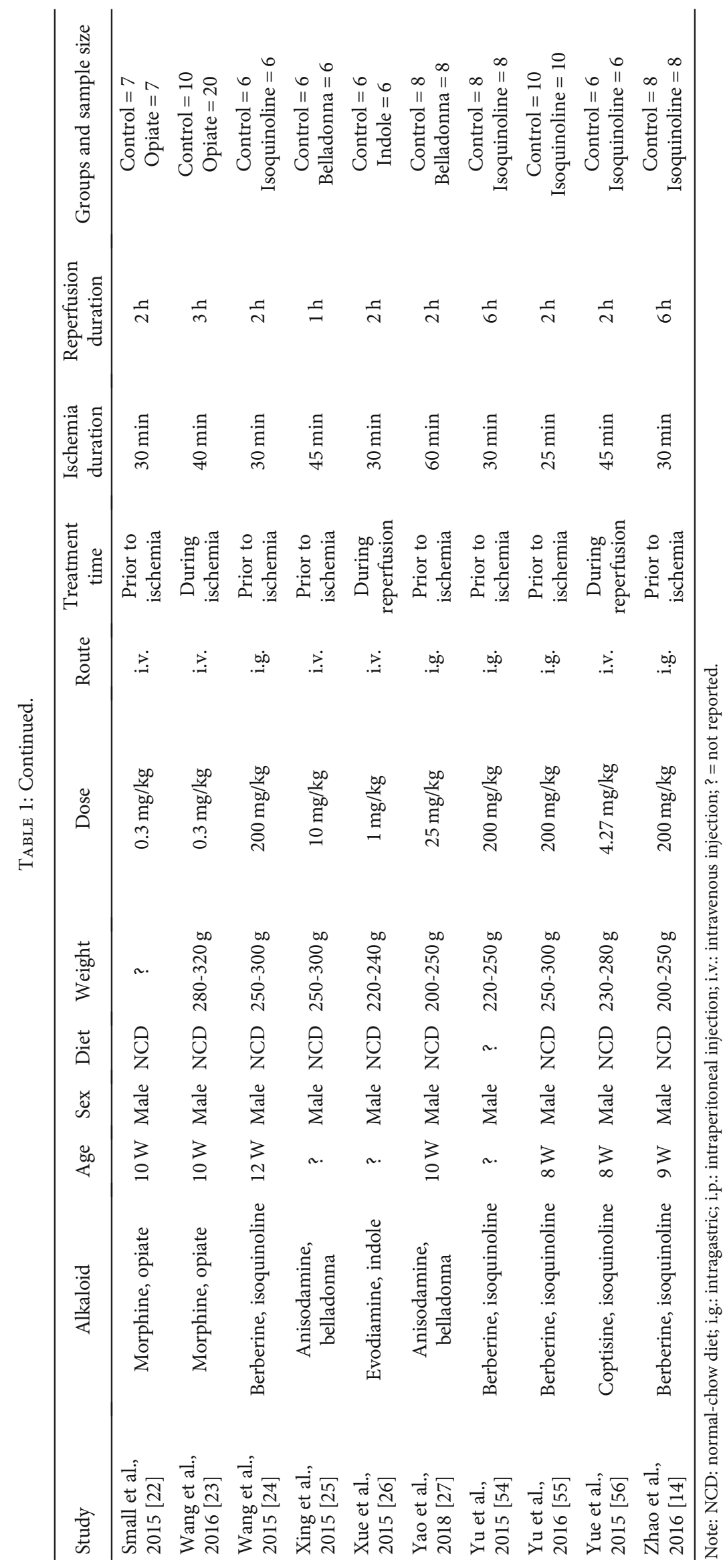




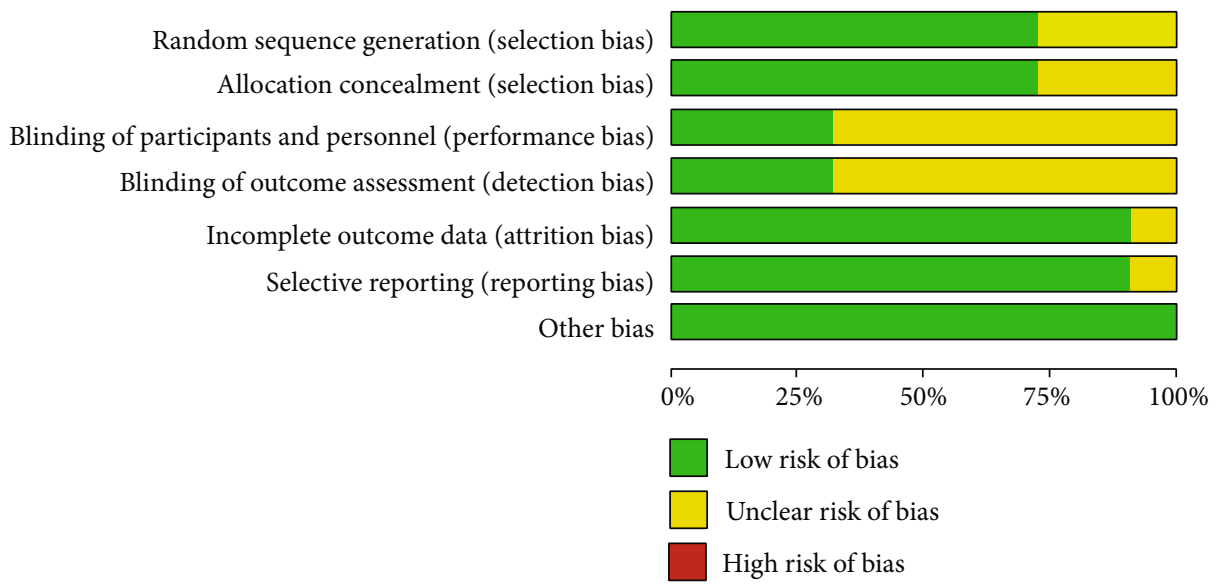

FIGURE 2: Risk of bias and quality assessment score (\%) per risk of bias item.

alkaloid doses were compared to a single control group. To avoid an artificial increase in sample size in the pooled analysis, the number of animals in the control group for each study was divided by the number of comparator groups.

Subgroup analyses were performed to evaluate the effects of aporphine, belladonna, indole, isoquinoline, opiate, and other alkaloids on infarct size. Sensitivity analyses were performed to determine whether the findings were robust. $P<0.05$ was considered statistically significant.

\section{Results}

3.1. Study Selection. In total, 1056 articles were identified in original search. After screening titles and abstracts, 40 studies were considered potentially eligible for inclusion. After estimating full-text publications, 14 studies were excluded due to intervention or outcome data were unqualified [28-41], and four studies were excluded because pure alkaloids were not assessed [42-45]. Finally, 22 studies were included in the present meta-analysis [13-14, 19-27, 46-56] (Figure 1).

3.2. Study Characteristics. The included studies contained 35 datasets, a total of 513 animals, and the characteristics are described in Table 1. The intervention was isoquinoline alkaloids in 10 studies [13-14, 19, 24, 47-48, 50,54-56], opiate alkaloids in four studies [20-23], indole alkaloids in two studies [26, 53], aporphine alkaloids in two studies [46, 49], belladonna alkaloids in two studies [25, 27], and other alkaloids in two studies $[51,52]$.

Twenty-one studies used male animals [13, 14, 19-27, $46-50,52-56]$, and the gender of the animals was not reported in one study [51]. Rats received a normal chow diet in 17 studies $[13,14,19,20,22-27,47,49-52,55,56]$, and the diet was not reported in five studies $[21,46,48,53,54]$. Alkaloid administration was initiated in 8-week-old rats in five studies $[19,49,51,55,56]$, 9-week-old rats in two studies $[14,48], 10$-week-old rats in six studies [13, 21-23, 27, 52], and 12-week-old rats in one study [24], and animal age was not reported in eight studies [20, 25, 26, 46, 47, 50, 53, 54]. The weight of the rats varied from 200 to $350 \mathrm{~g}$.

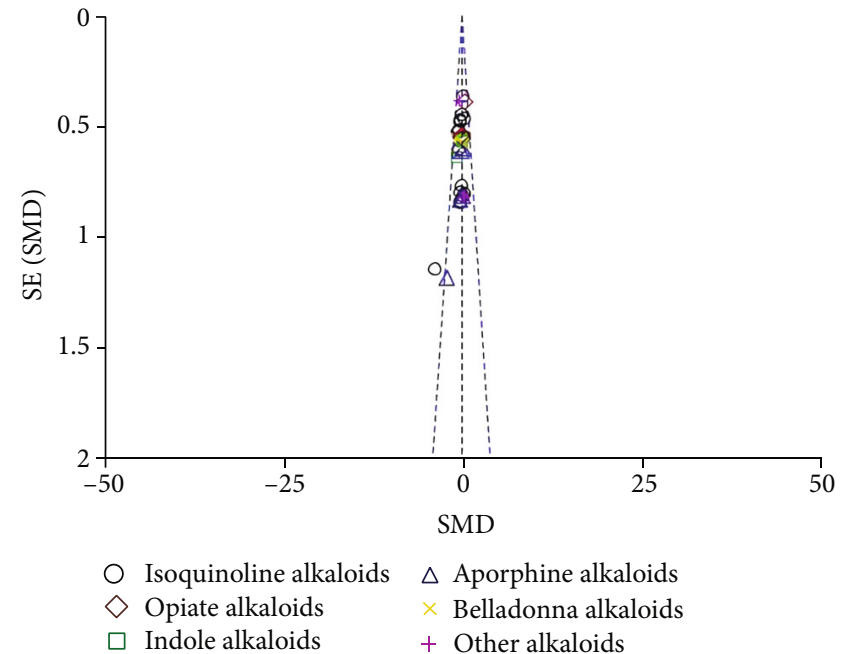

FIgURe 3: A funnel plot for evaluating publication bias.

The route of alkaloid administration was intravenous in 11 studies [20-23, 25, 26, 46, 49, 51, 53, 56], intragastric in nine studies $[13,14,19,24,27,47,52,54,55]$, and intraperitoneal in two studies $[48,50]$. The duration of ischemia varied from 30 to $60 \mathrm{~min}$, and that of reperfusion varied from $30 \mathrm{~min}$ to $24 \mathrm{~h}$. Treatment was conducted prior to ischemia in 17 studies [13, 14, 19-22, 24, 25, 27, 47-52, 54, 55] and during ischemia in five studies $[23,26,46,53,56]$.

3.3. Quality Assessment. The results of the study quality assessment are presented in Figure 2. In total, 16 studies were randomized. The risks of bias caused allocation concealment, and blinding was 72.7 and $31.8 \%$, respectively. It showed no missing outcome data in twenty studies, and the risk of selective outcomes reporting was unclear in two studies. Across studies, the risk of bias from other sources was low. Visual inspection of the funnel plot indicated substantial publication bias (Figure 3).

3.4. Effect of Alkaloids on Infarct Size. The effect of alkaloids on infarct size was reported for 35 datasets obtained from 22 studies (alkaloid group, $n=325$; vehicle group, $n=188$ ). 


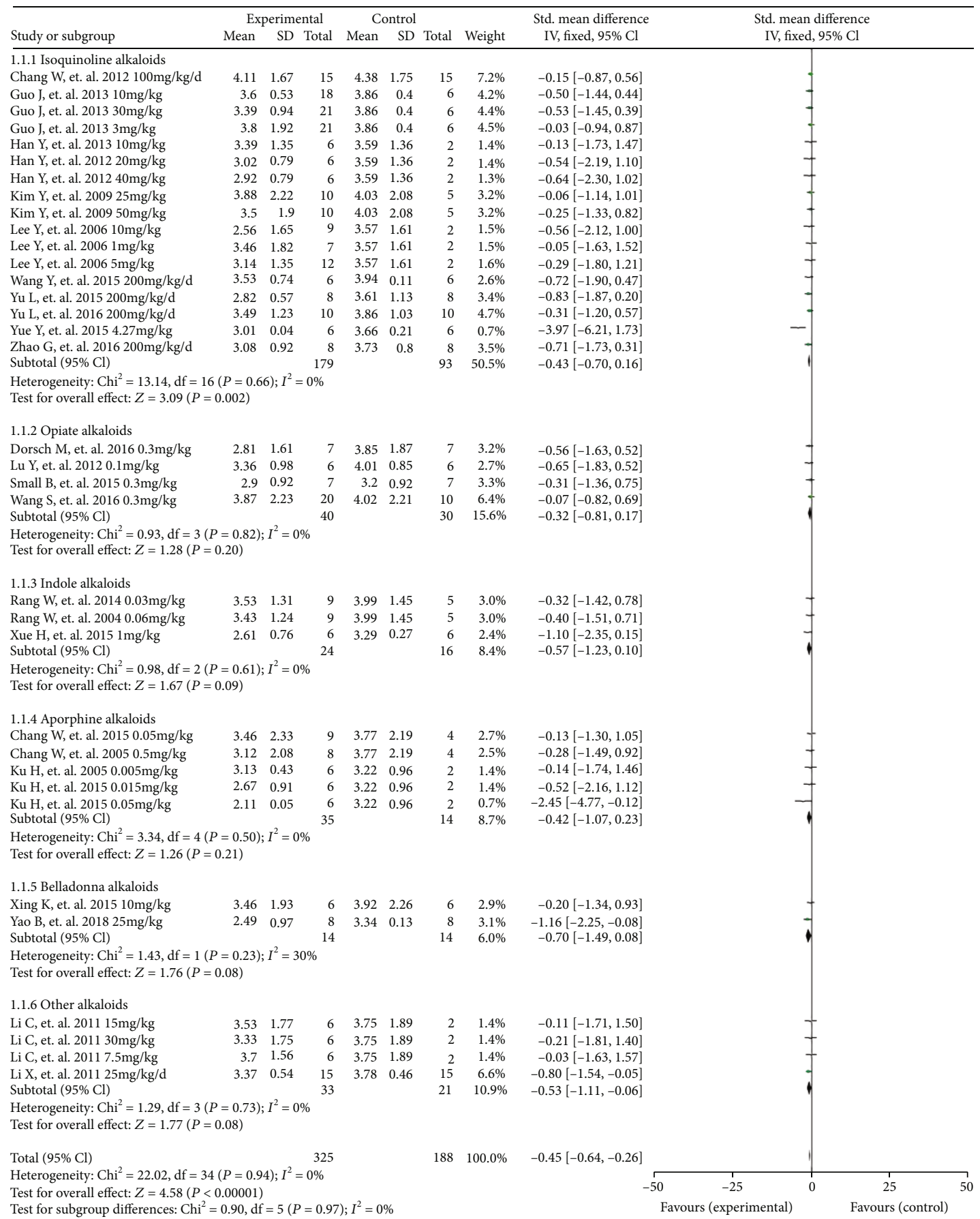

FIGURE 4: A forest plot of the effects of alkaloids on infarction size. Subgroup analyses evaluated the effects of isoquinoline, opiate, indole, aporphine, belladonna, and other alkaloids. SD: standard deviation; CI: confidence interval; Std: standard; IV: inverse variance.

The meta-analysis indicated that compared with the effects of vehicle, alkaloids significantly reduced infarct size $(P<0.00001 ; \mathrm{SMD}=-0.45 ; 95 \% \mathrm{CI}=-0.64$ to -0.26$)$. There was no evidence of heterogeneity between studies $\left(I^{2}=0 \%\right.$, Figure 4).

Subgroup analyses were performed to evaluate the effects of isoquinoline, opiate, indole, aporphine, belladonna, and other alkaloids on infarct size. Isoquinoline alkaloids (alkaloid group, $n=179$; vehicle group, $n=93$ ) significantly reduced infarct size versus vehicle $(P=0.002 ; \mathrm{SMD}=-0.43$; $95 \% \mathrm{CI}=-0.70$ to -0.16$)$, and there was no evidence of heterogeneity between studies $\left(I^{2}=0 \%\right)$. Opiate alkaloids (alkaloid group, $n=40$; vehicle group, $n=30 ; P=0.20$; $\mathrm{SMD}=-0.32 ; 95 \% \mathrm{CI}=-0.81$ to 0.17 ), indole alkaloids (alkaloid group, $n=24$; vehicle group, $n=16 ; P=0.09$; $\mathrm{SMD}=-0.57 ; 95 \% \mathrm{CI}=-1.23$ to 0.10 ), aporphine alkaloids (alkaloid group, $n=35$; vehicle group, $n=14 ; P=0.21$; $\mathrm{SMD}=-0.42 ; 95 \% \mathrm{CI}=-1.07,0.23)$, and other alkaloids 


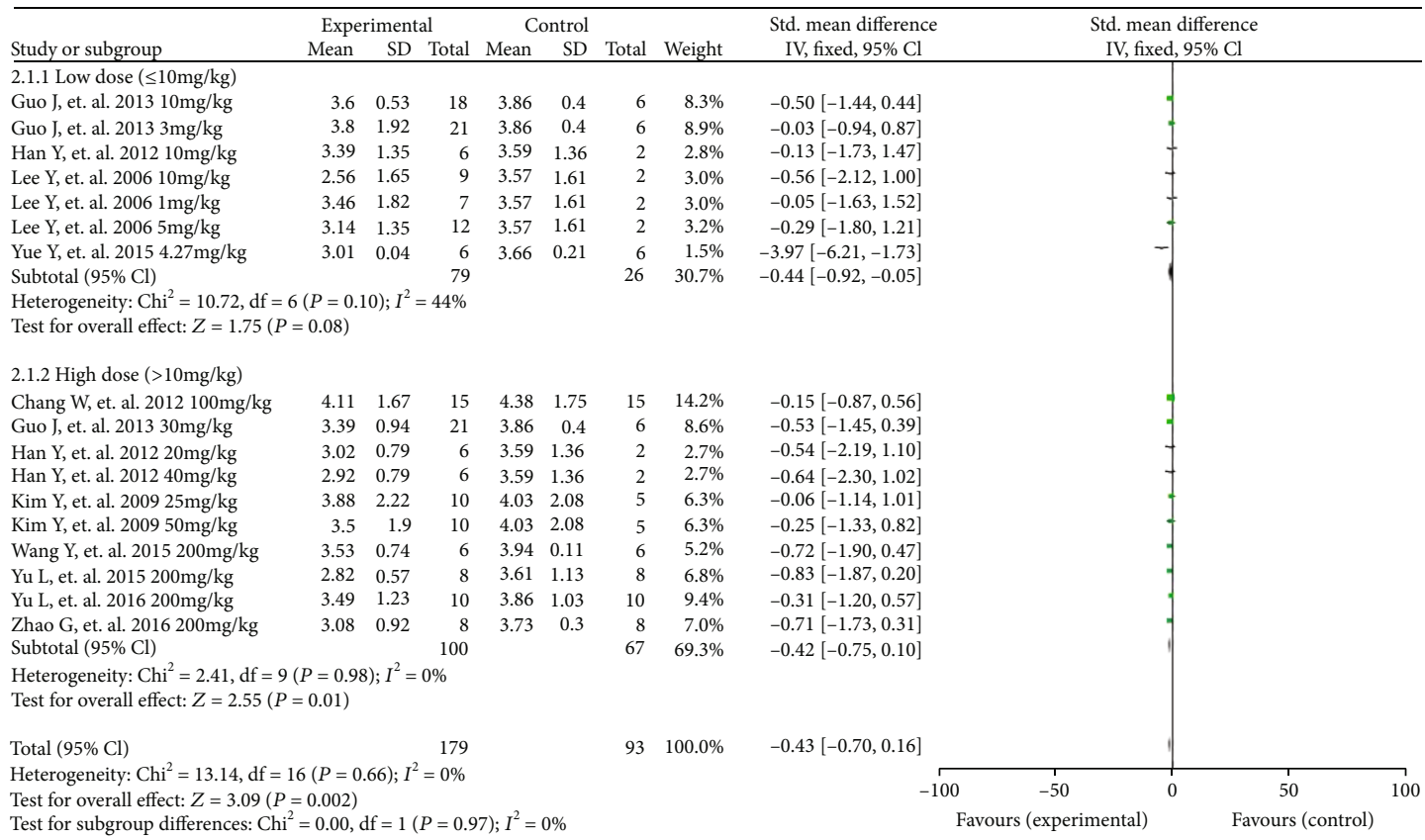

FIGURE 5: A forest plot of the effects of isoquinoline alkaloids on infarction size between high and low doses. SD: standard deviation; CI: confidence interval; Std: standard; IV: inverse variance.

(alkaloid group, $n=33$; vehicle group, $n=21 ; P=0.08$; $\mathrm{SMD}=-0.53 ; 95 \% \mathrm{CI}=-1.11,0.06)$ did not significantly decrease infarct size compared with the effects of vehicle, and there was no evidence of heterogeneity between studies $\left(I^{2}=0 \%\right)$. Belladonna alkaloids (alkaloid group, $n=14$; vehicle group, $n=14$ ) did not significantly reduce infarct size versus the control $(P=0.08 ; \mathrm{SMD}=-0.70 ; 95 \% \mathrm{CI}=-1.49$ to $0.08)$, and there was a moderate degree of heterogeneity between studies $\left(I^{2}=30 \%\right)$.

In a subgroup analysis of isoquinoline alkaloids, highdose ( $>10 \mathrm{mg} / \mathrm{kg}$ ) treatment (alkaloid group, $n=100$; vehicle group, $n=100)$ significantly reduced infarct size compared with the effects of vehicle $(P=0.01 ; \mathrm{SMD}=-0.42 ; 95 \% \mathrm{CI}=$ -0.75 to -0.10$)$, and there was no evidence of heterogeneity between studies $\left(I^{2}=0 \%\right)$. Low-dose $(\leq 10 \mathrm{mg} / \mathrm{kg})$ treatment (alkaloid group, $n=79$; vehicle group, $n=26 ; P=0.08$; $\mathrm{SMD}=-0.44 ; 95 \% \mathrm{CI}=-0.92$ to 0.05$)$ also significantly reduced infarct size versus vehicle, but there was evidence of moderate heterogeneity between studies $\left(I^{2}=44 \%\right.$, Figure 5$)$.

The overall findings of the sensitivity analysis were not changed when a fixed-effects model was used instead of a random-effects model (SMD (95\% CI): $-0.45 \quad(-0.64$ to $-0.26)$ vs. $-0.55(-0.67$ to -0.43$)$ and $-0.43(-0.70$ to -0.16$)$ vs. $-0.55(-0.69$ to -0.42$))$.

\section{Discussion}

Based on previous animal studies, alkaloids have the potential to attenuate myocardial I/R injury. However, parameters such as the age and gender of animals, sample size, route type, and duration of ischemia and reperfusion varied among the studies. A quantitative synthesis and systematic analysis for animal study data that account for the sources of hetero- geneity may have a potential to demonstrate the clinically desirable therapeutic efficacy of alkaloids in alleviating myocardial I/R injury. So we performed this systematic review and meta-analysis to evaluate the effects of alkaloids on myocardial I/R injury in rats. The findings illustrated that alkaloids significantly reduced myocardial infarct size versus vehicle.

Several alkaloid structures exist, including pyridines, isoquinolines, quinolines, indoles, purines, and aporphines [57]. In subgroup analyses, isoquinoline alkaloids significantly reduced infarct size compared with the vehicle in rats with myocardial I/R injury.

To our knowledge, this systematic review and metaanalysis is the first such study to estimate the efficacy of alkaloids in alleviating myocardial I/R injury in rats. The findings are potential to demonstrate a scientific basis for clinical studies of alkaloids in the treatment of myocardial I/R injury.

There was no heterogeneity between studies in the overall analysis, but there was a moderate degree of heterogeneity between studies in the analysis of belladonna alkaloids. Potential sources of heterogeneity include the duration of ischemia and reperfusion, each of which can progressively increase infarct size, and heterogeneity was found to be independently associated with both parameters [58].

This meta-analysis was associated with some limitations. First, it is necessary to discuss the relevance of our findings to humans due to species-specific differences in myocardial physiology. The rat myocardial I/R injury model is well established for studying myocardial ischemic disease. However, there are differences in pathogenesis. Specifically, the coronary arteries include the left anterior descending, circumflex, and right coronary arteries. All of these vessels can be attacked in patients with myocardial ischemic disease. 
However, only the left anterior descending artery was considered across studies. Second, only one parameter, infarct size, was used to estimate the efficacy of alkaloids in alleviating myocardial I/R injury. Other parameters, such as creatine kinase, cardiac troponin, heart rate, blood pressure, left ventricular developed pressure, and left ventricular end-diastolic pressure, were not considered [59-61]. Third, in this metaanalysis, other animal models of myocardial ischemiareperfusion injury, such as mice, rabbits, and pigs, were not included. Fourth, the sample sizes of some included studies were relatively small. Further evaluation of animal studies with larger sample sizes is required to certify whether alkaloids are beneficial for alleviating myocardial I/R injury in humans.

The present meta-analysis and systematic review indicated that isoquinoline alkaloids have a clinical benefit on myocardial I/R injury in rats. Therefore, large scale, prospective, and well-designed animal studies are necessary to validate the mechanism of alkaloids in alleviating myocardial $\mathrm{I} / \mathrm{R}$ injury. And more randomized controlled trials in humans are needed before the pharmacological therapeutic effects on myocardial I/R injury of alkaloids in patients are confirmed.

\section{Data Availability}

The data used and/or analyzed during the current study are available from the corresponding author on reasonable request.

\section{Conflicts of Interest}

The authors declare that they have no competing interests.

\section{Authors' Contributions}

SW conducted the analysis and wrote the manuscript; HL and YZ collected and performed a preliminary analysis of references; LR revised the manuscript and approved it for submission.

\section{Acknowledgments}

This work was supported by the National Natural Science Foundation of China (No. 81773934).

\section{References}

[1] Z. H. Geng, L. Huang, M. B. Song, and Y. M. Song, "Protective effect of a polysaccharide from Salvia miltiorrhiza on isoproterenol (ISO)-induced myocardial injury in rats," Carbohydrate Polymers, vol. 132, pp. 638-642, 2015.

[2] K. Mnafgui, R. Hajji, F. Derbali et al., "Protective effect of hydroxytyrosol against cardiac remodeling after isoproterenolinduced myocardial infarction in rat," Cardiovascular Toxicology, vol. 16, no. 2, pp. 147-155, 2016.

[3] E. Braunwald and R. A. Kloner, "Myocardial reperfusion: a double-edged sword?," The Journal of Clinical Investigation, vol. 76, no. 5, pp. 1713-1719, 1985.
[4] Y. J. Zhang, S. H. Yang, M. H. Li et al., "Berberine attenuates adverse left ventricular remodeling and cardiac dysfunction after acute myocardial infarction in rats: role of autophagy," Clinical and Experimental Pharmacology \& Physiology, vol. 41, no. 12, pp. 995-1002, 2014.

[5] G. Paradies, V. Paradies, F. M. Ruggiero, and G. Petrosillo, "Cardiolipin alterations and mitochondrial dysfunction in heart ischemia/reperfusion injury," Clin. Lipidol., vol. 10, no. 5, pp. 415-429, 2015.

[6] Y. Zhang, X. J. Ma, C. Y. Guo et al., "Pretreatment with a combination of ligustrazine and berberine improves cardiac function in rats with coronary microembolization," Acta Pharmacologica Sinica, vol. 37, no. 4, pp. 463-472, 2016.

[7] Y. Bai, Z. Li, W. Liu, D. Gao, M. Liu, and P. Zhang, "Biochanin A attenuates myocardial ischemia/reperfusion injury through the TLR4/NF- $\kappa \mathrm{B} / \mathrm{NLRP} 3$ signaling pathway," Acta Cirúrgica Brasileira, vol. 34, no. 11, p. e201901104, 2019.

[8] J. Zhang, Y. Zhou, Y. Sun et al., "Beneficial effects of oridonin on myocardial ischemia/reperfusion injury: insight gained by metabolomic approaches," European Journal of Pharmacology, vol. 861, p. 172587, 2019.

[9] Y. Zhang, M. Li, X. Li, T. Zhang, M. Qin, and L. Ren, "Isoquinoline alkaloids and indole alkaloids attenuate aortic atherosclerosis in apolipoprotein E deficient mice: a systematic review and meta-analysis," Frontiers in Pharmacology, vol. 9, p. 602, 2018.

[10] S. Li, S. J. Priceman, H. Xin et al., "Icaritin inhibits JAK/STAT3 signaling and growth of renal cell carcinoma," PLoS One, vol. 8, no. 12, article e81657, 2013.

[11] M. Y. Jun, R. Karki, K. R. Paudel, B. R. Sharma, D. Adhikari, and D. W. Kim, "Alkaloid rich fraction from Nelumbo nucifera targets VSMC proliferation and migration to suppress restenosis in balloon-injured rat carotid artery," Atherosclerosis, vol. 248, pp. 179-189, 2016.

[12] M. A. Ansari, A. Iqubal, R. Ekbbal, and S. E. Haque, "Effects of nimodipine, vinpocetine and their combination on isoproterenol-induced myocardial infarction in rats," Biomedicine \& Pharmacotherapy, vol. 109, pp. 1372-1380, 2019.

[13] J. Guo, S. B. Wang, T. Y. Yuan et al., "Coptisine protects rat heart against myocardial ischemia/reperfusion injury by suppressing myocardial apoptosis and inflammation," Atherosclerosis, vol. 231, no. 2, pp. 384-391, 2013.

[14] G. L. Zhao, L. M. Yu, W. L. Gao et al., "Berberine protects rat heart from ischemia/reperfusion injury via activating JAK2/STAT3 signaling and attenuating endoplasmic reticulum stress," Acta Pharmacologica Sinica, vol. 37, no. 3, pp. 354-367, 2016.

[15] Z. Xu, J. Alloush, E. Beck, and N. Weisleder, “A murine model of myocardial ischemia-reperfusion injury through ligation of the left anterior descending artery," Journal of Visualized Experiments, vol. 86, 2014.

[16] C. R. Hooijmans and M. Ritskes-Hoitinga, "Progress in using systematic reviews of animal studies to improve translational research," PLoS Medicine, vol. 10, no. 7, article e1001482, 2013.

[17] R. B. M. de Vries, C. R. Hooijmans, M. W. Langendam et al., "A protocol format for the preparation, registration and publication of systematic reviews of animal intervention studies," Evidence-based Preclinical Medicine, vol. 2, no. 1, 2015.

[18] C. R. Hooijmans, M. M. Rovers, R. B. de Vries, M. Leenaars, M. Ritskes-Hoitinga, and M. W. Langendam, "SYRCLE's risk of bias tool for animal studies," BMC Medical Research Methodology, vol. 14, no. 1, 2014. 
[19] W. Chang, M. Zhang, J. Li et al., "Berberine attenuates ischemia-reperfusion injury via regulation of adenosine- $5^{\prime}$ -monophosphate kinase activity in both non-ischemic and ischemic areas of the rat heart," Cardiovascular Drugs and Therapy, vol. 26, no. 6, pp. 467-478, 2012.

[20] M. Dorsch, F. Behmenburg, M. Raible et al., "Morphineinduced preconditioning: involvement of protein kinase A and mitochondrial permeability transition pore," PLoS One, vol. 11, no. 3, 2016.

[21] Y. Lu, C. S. Dong, J. M. Yu, and H. Li, "Morphine reduces the threshold of remote ischemic preconditioning against myocardial ischemia and reperfusion injury in rats: the role of opioid receptors," Journal of Cardiothoracic and Vascular Anesthesia, vol. 26, no. 3, pp. 403-406, 2012.

[22] B. A. Small, Y. Lu, A. K. Hsu, G. J. Gross, and E. R. Gross, "Morphine reduces myocardial infarct size via heat shock protein 90 in rodents," BioMed Research International, vol. 2015, Article ID 129612, 8 pages, 2015.

[23] S. Y. Wang, X. L. Cui, F. S. Xue et al., "Combined morphine and limb remote ischemic perconditioning provides an enhanced protection against myocardial ischemia/reperfusion injury by antiapoptosis," The Journal of Surgical Research, vol. 202, no. 1, pp. 13-25, 2016.

[24] Y. Wang, J. Liu, A. Ma, and Y. Chen, "Cardioprotective effect of berberine against myocardial ischemia/reperfusion injury via attenuating mitochondrial dysfunction and apoptosis," International Journal of Clinical and Experimental Medicine, vol. 8, no. 8, pp. 14513-14519, 2015.

[25] K. Xing, X. Fu, L. Jiang et al., "Cardioprotective effect of anisodamine against myocardial ischemia injury and its influence on cardiomyocytes apoptosis," Cell Biochemistry and Biophysics, vol. 73, no. 3, pp. 707-716, 2015.

[26] H. Xue, Y. Cheng, X. Wang, Y. Yue, W. Zhang, and X. Li, "Rutaecarpine and evodiamine selected as $\beta_{1}$-AR inhibitor candidates using $\beta_{1}$-AR/CMC-offline-UPLC/MS prevent cardiac ischemia-reperfusion injury via energy modulation," Journal of Pharmaceutical and Biomedical Analysis, vol. 115, pp. 307-314, 2015.

[27] B. J. Yao, X. Q. He, Y. H. Lin, and W. J. Dai, “Cardioprotective effects of anisodamine against myocardial ischemia/reperfusion injury through the inhibition of oxidative stress, inflammation and apoptosis," Molecular Medicine Reports, vol. 17, no. 1, pp. 1253-1260, 2018.

[28] X. C. Yu, S. Wu, G. Y. Wang et al., "Cardiac effects of the extract and active components of radix Stephaniae tetrandrae. II. Myocardial infarct, arrhythmias, coronary arterial flow and heart rate in the isolated perfused rat heart," Life Sciences, vol. 68, no. 25, pp. 2863-2872, 2001.

[29] C. P. Hu, N. S. Li, L. Xiao, H. W. Deng, and Y. J. Li, "Involvement of capsaicin-sensitive sensory nerves in cardioprotection of rutaecarpine in rats," Regulatory Peptides, vol. 114, no. 1, pp. 45-49, 2003.

[30] L. Groban, J. C. Vernon, and J. Butterworth, "Intrathecal morphine reduces infarct size in a rat model of ischemiareperfusion injury," Anesthesia and Analgesia, vol. 98, no. 4, pp. 903-909, 2004.

[31] Z. Chen, T. Li, and B. Zhang, "Morphine postconditioning protects against reperfusion injury in the isolated rat hearts," The Journal of Surgical Research, vol. 145, no. 2, pp. 287-294, 2008.

[32] J. L. Ling, G. T. Wong, L. Yao, Z. Xia, and M. G. Irwin, "Remote pharmacological post-conditioning by intrathecal morphine: cardiac protection from spinal opioid receptor activation," Acta Anaesthesiologica Scandinavica, vol. 54, no. 9, pp. 1097-1104, 2010.

[33] L. Li, H. Zhang, T. Li, and B. Zhang, "Involvement of adenosine monophosphate-activated protein kinase in morphineinduced cardioprotection," The Journal of Surgical Research, vol. 169, no. 2, pp. 179-187, 2011.

[34] C. M. Zhang, L. Gao, Y. J. Zheng, and H. T. Yang, "Berbamine protects the heart from ischemia/reperfusion injury by maintaining cytosolic $\mathrm{Ca}(2+)$ homeostasis and preventing calpain activation," Circulation Journal, vol. 76, no. 8, pp. 19932002, 2012.

[35] Y. Lu, G. T. C. Wong, Y. Zhang, J. Hu, and C. Dong, "Remote intrathecal morphine preconditioning is ineffective in the presence of neuraxial blockade with lidocaine," The Kaohsiung Journal of Medical Sciences, vol. 30, no. 2, pp. 68-72, 2014.

[36] Y. Lu, J. Hu, Y. Zhang, C. S. Dong, and G. T. C. Wong, "Remote intrathecal morphine preconditioning confers cardioprotection via spinal cord nitric oxide/cyclic guanosine monophosphate/protein kinase G pathway," The Journal of Surgical Research, vol. 193, no. 1, pp. 43-51, 2015.

[37] Z. Chen, D. R. Spahn, X. Zhang, Y. Liu, H. Chu, and Z. Liu, "Morphine postconditioning protects against reperfusion injury: the role of protein kinase C-epsilon, extracellular signal-regulated kinase $1 / 2$ and mitochondrial permeability transition pores," Cellular Physiology and Biochemistry, vol. 39, no. 5, pp. 1930-1940, 2016.

[38] X. Cheng, Y. E. Zhang, X. Lu, Y. Lu, and Z. Chen, "The involvement of central beta-endorphin in the cardioprotective effects of remote preconditioning mediated by the intracerebroventricular administration of morphine," Irish Journal of Medical Science, vol. 185, no. 2, pp. 423-431, 2016.

[39] H. He, J. Huh, H. Wang, Y. Kang, J. Lou, and Z. Xu, "Mitochondrial events responsible for morphine's cardioprotection against ischemia/reperfusion injury," Toxicology and Applied Pharmacology, vol. 290, pp. 66-73, 2016.

[40] I. Webster, A. Smith, A. Lochner, and B. Huisamen, “The role of MKP-1 in insulin-induced cardioprotection," Cardiovascular Drugs and Therapy, vol. 31, no. 3, pp. 247-254, 2017.

[41] Z. Chen, R. Liu, Q. Niu, H. Wang, Z. Yang, and Y. Bao, "Morphine postconditioning alleviates autophage in ischemiareperfusion induced cardiac injury through up-regulating lnc RNA UCA1," Biomedicine \& Pharmacotherapy, vol. 108, pp. 1357-1364, 2018.

[42] A. J. Zatta, H. Kin, D. Yoshishige et al., "Evidence that cardioprotection by postconditioning involves preservation of myocardial opioid content and selective opioid receptor activation," American Journal of Physiology. Heart and Circulatory Physiology, vol. 294, no. 3, pp. 1444-1451, 2008.

[43] S. Mimuro, T. Katoh, A. Suzuki et al., "Deterioration of myocardial injury due to dexmedetomidine administration after myocardial ischaemia," Resuscitation, vol. 81, no. 12, pp. 1714-1717, 2010.

[44] Y. C. Xu, R. P. Li, F. S. Xue et al., " $\kappa$-Opioid receptors are involved in enhanced cardioprotection by combined fentanyl and limb remote ischemic postconditioning," Journal of Anesthesia, vol. 29, no. 4, pp. 535-543, 2015.

[45] Q. Liu, Z. Li, Y. Liu et al., "Hydromorphine postconditioning protects isolated rat heart against ischemia-reperfusion injury via activating P13K/Akt/eNOS signaling," Cardiovascular Therapeutics, vol. 36, no. 6, 2019. 
[46] W. L. Chang, S. S. Lee, and M. J. Su, “Attenuation of postischemia reperfusion injury by thaliporphine and morphine in rat hearts," Journal of Biomedical Science, vol. 12, no. 4, pp. 611-619, 2005.

[47] Y. Han, W. Zhang, Y. Tang et al., "l-Tetrahydropalmatine, an active component of Corydalis yanhusuo W.T. Wang, protects against myocardial ischaemia-reperfusion injury in rats," PLoS One, vol. 7, no. 6, 2012.

[48] Y. M. Kim, Y. M. Ha, Y. C. Jin et al., "Palmatine from Coptidis rhizoma reduces ischemia-reperfusion-mediated acute myocardial injury in the rat," Food and Chemical Toxicology, vol. 47, no. 8, pp. 2097-2102, 2009.

[49] H. C. Ku, S. Y. Lee, C. H. Chen et al., “TM-1-1DP exerts protective effect against myocardial ischemia reperfusion injury via AKT-eNOS pathway," Naunyn-Schmiedeberg's Archives of Pharmacology, vol. 388, no. 5, pp. 539-548, 2015.

[50] Y. S. Lee, Y. J. Kang, H. J. Kim et al., "Higenamine reduces apoptotic cell death by induction of heme oxygenase-1 in rat myocardial ischemia-reperfusion injury," Apoptosis, vol. 11, no. 7, pp. 1091-1100, 2006.

[51] C. Li, Y. Gao, J. Tian, J. Shen, Y. Xing, and Z. Liu, “Sophocarpine administration preserves myocardial function from ischemia-reperfusion in rats via NF- $\kappa$ B inactivation," Journal of Ethnopharmacology, vol. 135, no. 3, pp. 620-625, 2011.

[52] X.-Y. Li, L. Xu, G.-S. Lin et al., "Protective effect of caffeine administration on myocardial ischemia/reperfusion injury in rats," Shock, vol. 36, no. 3, pp. 289-294, 2011.

[53] W.-Q. Rang, Y.-H. Du, C.-P. Hu et al., "Protective effects of evodiamine on myocardial ischemia-reperfusion injury in rats," Planta Medica, vol. 70, no. 12, pp. 1140-1143, 2004.

[54] L. Yu, F. Li, G. Zhao et al., "Protective effect of berberine against myocardial ischemia reperfusion injury: role of Notch 1/Hes1-PTEN/Akt signaling," Apoptosis, vol. 20, no. 6, pp. 796-810, 2015.

[55] L. Yu, Q. Li, B. Yu et al., "Berberine attenuates myocardial ischemia/reperfusion injury by reducing oxidative stress and inflammation response: role of silent information regulator 1," Oxidative Medicine and Cellular Longevity, vol. 2016, Article ID 1689602, 16 pages, 2016.

[56] Y. Yue, L. Dou, X. Wang, H. Xue, Y. Song, and X. Li, “Screening $\beta_{1} \mathrm{AR}$ inhibitors by cell membrane chromatography and offline UPLC/MS method for protecting myocardial ischemia," Journal of Pharmaceutical and Biomedical Analysis, vol. 115, pp. 339-344, 2015.

[57] R. Eguchi, N. Ono, A. Hirai Morita et al., "Classification of alkaloids according to the starting substances of their biosynthetic pathways using graph convolutional neural networks," BMC Bioinformatics, vol. 20, no. 1, p. 380, 2019.

[58] X. Rossello, A. R. Hall, R. M. Bell, and D. M. Yellon, "Characterization of the Langendorff perfused isolated mouse heart model of global ischemia-reperfusion injury: impact of ischemia and reperfusion length on infarct size and LDH release," Journal of Cardiovascular Pharmacology and Therapeutics, vol. 21, no. 3, pp. 286-295, 2015.

[59] U. L. Choi, J. H. Park, B. J. Sun et al., "Impaired left ventricular diastolic function is related to the formation of left ventricular apical thrombus in patients with acute anterior myocardial infarction," Heart and Vessels, vol. 33, no. 5, pp. 447-452, 2018.
[60] Y. Sandoval, K. Sharain, A. K. Saenger, S. W. Smith, F. S. Apple, and A. S. Jaffe, "Clinical use of cardiac troponin for acute cardiac care and emerging opportunities in the outpatient setting," Minerva Medica, vol. 110, no. 2, pp. 139-156, 2019.

[61] M. Spindler, K. Meyer, H. Strömer et al., "Creatine kinasedeficient hearts exhibit increased susceptibility to ischemiareperfusion injury and impaired calcium homeostasis," American Journal of Physiology. Heart and Circulatory Physiology, vol. 287, no. 3, pp. H1039-H1045, 2004. 